2010;68:762-766). (Respond: Dr Joshua L Bonkowski, Dept Pediatrics, University of Utah Health Sciences Center, 295 Chipeta Way, Salt Lake City, UT 84108. E-mail: Joshua.bonkowski@hsc.utah.edu).

COMMENT. Pandemic $2009 \mathrm{H} 1 \mathrm{Nl}$ influenza is associated with heightened neurological complications in children compared to seasonal influenza.

In a study of 345 children hospitalized with 2009 influenza A (H1N1) in California, $30(8.7 \%)$ had CNS complications, including seizures $(\mathrm{n}=17)$, altered mental status/delirium (18), or both (12). Presentation with seizures $(\mathrm{p}=.01)$ or altered mental status $(\mathrm{p}<.001)$ was significantly associated with ICU admission or death. Eleven $(37 \%)$ of the 30 had preexisting cerebral palsy/developmental delay or seizure disorder. Seventeen (57\%) were admitted to the ICU. Median length of stay was 4 days. Of 8 fatal cases, 5 had comorbid neurological disorders, including 4 with cerebral palsy/developmental delay. (Louie JK, Gavali S, Acosta M, et al. Arch Pediatr Adolesc Med 2010;164(11):1023-1031).

In a study of 478 children with 2009 influenza A (H1N1) virus in Israel, 42 patients $(8.8 \%)$ were admitted to the PICU, and $41(8.6 \%)$ had seizures, mostly febrile. Patients with metabolic and neurological disorders were at highest risk for severe complications.(Stein M, Tasher D, Glikman D, et al. Arch Pediatr Adolesc Med 2010;164(11):1015-1022).

Influenza A-induced acute autonomic neuropathy is reported in a 15-year-old girl who developed severe and persistent orthostatic hypotension with loss of consciousness on standing. (Lukkarinen H, Peltola V. Pediatr Neurol Dec 2010;43:425-426). Removal of circulating autoantibodies with a single iv immunoglobulin dose $(2 \mathrm{~g} / \mathrm{kg})$ resulted in immediate recovery.

\title{
VARICELLA RETINITIS AND OPTIC NEURITIS
}

A 3-year-old immunocompetent boy with a 3-day history of typical chicken pox presented with blurred vision in the left eye, in a report from the University of Bern, Switzerland. The optic nerve was swollen and a puffy yellowish lesion covered the posterior pole and macula, consistent with retinitis and retinal edema. Active encephalitis was ruled out by lumbar puncture and negative CSF viral studies. Serology was positive for varicella (IgM), and negative for other virus infections. Treatment included a 10-day course of iv acyclovir and oral prednisone, followed by a 3 month course of oral acyclovir. The right eye was unaffected but a macular scar and optic nerve atrophy developed in the left eye, and visual acuity was 20/400 with no improvement after 9-year follow-up. (Tappeiner C, Aebi C, Garweg JG. Retinitis and optic neuritis in a child with chickenpox. Case report and review of literature. Pediatr Inf Dis Jrnl Dec 2010;29(12):1150-1152).(Respond: Justus G Garweg MD, Swiss Eye Institute, Bremgartenstrasse 119, 3012 Bern, Switzerland. E-mail: justus.garweg@swiss-eyeinstitute.com).

COMMENT. The authors cite only 5 cases of chickenpox associated retinitis and 10 of optic neuritis previously reported. Prompt ophthalmological examination is 
recommended in a child with varicella who presents with reddening of an eye or blurred vision, to exclude papillitis or necrotizing retinitis. Prompt antiviral therapy may help to preserve some residual vision and prevent extension of the lesion. Systemic corticosteroids are not generally advocated, and possible benefits of a brief course used in the above child with a fulminant retinopathy would require confirmation by controlled trial.

\section{HERPES SIMPLEX VIRUS REACTIVATION AFTER HEMISPHERECTOMY FOR INTRACTABLE SEIZURES}

Researchers at the Cleveland Clinic report a 23-month-old immunocompetent boy with reactivated herpes simplex virus encephalitis (HSE) after a subtotal hemispherectomy for seizures related to HSE-associated right middle cerebral artery infarction at $\bar{i}$ months of age. The diagnosis had been confirmed by HSV PCR from CSF, and he had completed a 21 day couse of iv acyclovir. Acyclovir and dexamethasone were administered routinely at time of surgery. Fever developed on postoperative day 1 and persisted despite continued acyclovir. CSF HSV PCR was positive for type 1 on postop day 8 and negative day 11 . At 3 weeks he was doing well with no seizures and was afebrile. Resected tissue showed multiple infarctions and microglial nodules, consistent with HSE history. (Gong T, Bingaman W, Danziger-Isakov L, Tuxhorn I, Goldfarb J. Herpes simplex virus reactivation after subtotal hemispherectomy in a pediatric patient. Pediatr Inf Dis Jrnl Dec 2010;29(12):1148-1150). (Respond:Johanna Goldfarb MD, 9500 Euclid Ave, S25, Cleveland, OH 44195. E-mail: goldfaj@ccf.org).

COMMENT. Reactivation of HSV after HSE is unusual and typically occurs immediately after treatment of a primary encephalitis. Cases with shorter courses of acyclovir may be susceptible. The authors cite one previous case in the literature of HSV reactivation after epilepsy surgery for mesial temporal sclerosis. The primary infection occurred at 16 months of age and reactivation 6 years later after hippocampectomy and gyrus corticotomy, the previous site of herpes infcction. (Bourgeois $\mathrm{M}$ et al. Neurosurgery 1999;44:633-635).

HSV reactivation should be ruled out by repeated CSF PCR in patients with persistent fever and irritability following epilepsy surgery. Transient immunosuppression complicating perioperative steroid therapy is suggested as a mechanism for HSV reactivation following hemispherectomy.

\section{DEMYELINATING DISORDERS}

\section{COGNITIVE IMPAIRMENT IN MULTIPLE SCLEROSIS AND MRI}

The utility of MRI techniques to monitor cognitive impairment progression in MS over time and to assess treatment is reviewed by researchers at University Hospital, San Raffaele, Milan, Italy; State University of New York, Buffalo; University of New Jersey, Newark; Leiden Institute for Brain and Cognition, the Netherlands; and University College and Institute of Neurology, Queen Square, London, UK. Focal white matter 